\title{
Correction to: Soluble CD163, adiponectin, C-reactive protein and progression of dysglycaemia in individuals at high risk of type 2 diabetes mellitus: the ADDITION-PRO cohort
}

\author{
Pia Deichgræber ${ }^{1}$ • Daniel R. Witte ${ }^{2,3} \cdot$ Holger J. Møller $^{4}$ • Mette V. Skriver ${ }^{5}$. \\ Bjørn Richelsen $^{6}$ • Marit E. Jørgensen ${ }^{7,8}$ • Nanna B. Johansen ${ }^{3,7,9} \cdot$ Annelli Sandbæk $^{1}$
}

Published online: 4 December 2017

(C) Springer-Verlag GmbH Germany, part of Springer Nature 2017

\section{Correction to: Diabetologia \\ https://doi.org/10.1007/s00125-016-4075-4}

The authors have discovered a coding error in the statistical analysis syntax file used for the mixed-effect model analyses in this paper. The error has led to differences (first decimal) in the estimates for the main results. Although the conclusions stand unaltered the actual change over time for many parameters is smaller than previously reported and the direction of change reversed for fasting insulin, HOMA-IR and HOMA- $\beta$.

The Abstract, Results, Discussion and Tables 2, 3 and 4 are reproduced here, with the changes shown in bold type. Amended ESM is also available. Please note that contrary to the statement in the original paper no individuals were excluded from the mixed-effect model analyses on CRP and $2 \mathrm{hPG}$.

The online version of the original article can be found under https://doi. org/10.1007/s00125-016-4075-4

Electronic supplementary material The online version of this article (https://doi.org/10.1007/s00125-017-4498-6) contains peer-reviewed but unedited supplementary material, which is available to authorised users.

Pia Deichgræber

p.deichgraeber@ph.au.dk

1 Section of General Practice, Department of Public Health, Aarhus University, Bartholins Allé 2, DK-8000 Aarhus, Denmark

2 Department of Public Health, Aarhus University, Aarhus, Denmark

3 Danish Diabetes Academy, Odense, Denmark

4 Department of Clinical Biochemistry, Aarhus University Hospital, Aarhus, Denmark

\begin{abstract}
Aim/hypothesis Our aim was to investigate the association between the macrophage-activation marker soluble CD163 (sCD163), adiponectin, C-reactive protein (CRP) and changes in glycaemia, insulin resistance and insulin secretion in individuals at high risk of type 2 diabetes mellitus.

Methods This prospective study included 1014 individuals at high risk of type 2 diabetes mellitus participating in the Danish arm of the Anglo-Danish-Dutch study of Intensive Treatment In PeOple with ScreeN-detected Diabetes in Primary Care (ADDITION-Europe trial) baseline examination in 20012006 and follow-up examination (ADDITION-Progression [ADDITION-PRO]) in 2009-2011. Baseline serum samples were analysed for sCD163, adiponectin and CRP. The
\end{abstract}

5 Section of Health Promotion and Health Services, Department of Public Health, Aarhus University, Aarhus, Denmark

6 Department of Endocrinology and Internal Medicine (MEA), Aarhus University Hospital, Aarhus, Denmark

7 Steno Diabetes Center, Gentofte, Denmark

8 National Institute of Public Health, University of Southern Denmark, Odense, Denmark

9 Research Centre for Prevention and Health, Centre for Health, Rigshospitalet-Glostrup, Glostrup, Denmark 
Table 2 Association of baseline sCD163 with glycaemia, insulin resistance and insulin secretion

\begin{tabular}{|c|c|c|c|c|c|c|c|}
\hline \multirow[t]{2}{*}{ Outcome } & \multirow[t]{2}{*}{$n$} & \multicolumn{2}{|l|}{ Model 1} & \multicolumn{2}{|l|}{ Model 2} & \multicolumn{2}{|l|}{ Model 3} \\
\hline & & $\beta(95 \% \mathrm{CI})$ & $p$ value & $\beta(95 \% \mathrm{CI})$ & $p$ value & $\beta(95 \% \mathrm{CI})$ & $p$ value \\
\hline \multicolumn{8}{|l|}{ Time-dependent } \\
\hline $\mathrm{HbA}_{1 \mathrm{c}}(\%)$ & 968 & $0.002(-0.004 ; 0.009)$ & 0.519 & $0.002(-0.004 ; 0.009)$ & 0.513 & $0.002(-0.004 ; 0.009)$ & 0.514 \\
\hline FPG (mmol/l) & 916 & $0.013(-0.002 ; 0.028)$ & 0.087 & $0.013(-0.002 ; 0.027)$ & 0.091 & $0.013(-0.002 ; 0.027)$ & 0.091 \\
\hline $2 \mathrm{hPG}(\mathrm{mmol} / \mathrm{l})$ & 601 & $0.030(-0.029 ; 0.088)$ & 0.322 & $0.029(-0.030 ; 0.089)$ & 0.328 & $0.029(-0.030 ; 0.089)$ & 0.328 \\
\hline Insulin (pmol/l) & 1001 & $1.078^{\mathrm{a}}(0.454 ; 1.702)$ & 0.001 & $1.076(0.452 ; 1.700)$ & 0.001 & $1.076(0.452 ; 1.700)$ & 0.001 \\
\hline HOMA-IR & 875 & $0.017(-0.012 ; 0.046)$ & 0.248 & $0.018(-0.011 ; 0.048)$ & 0.215 & $0.018(-0.011 ; 0.047)$ & 0.225 \\
\hline НOMA- $\beta$ & 875 & $1.313(0.537 ; 2.089)$ & 0.001 & $1.323(0.547 ; 2.099)$ & 0.001 & $1.323(0.547 ; 2.099)$ & 0.001 \\
\hline \multicolumn{8}{|l|}{ Time-independent } \\
\hline $\mathrm{HbA}_{1 \mathrm{c}}(\%)$ & 968 & $-0.015(-0.065 ; 0.034)$ & 0.540 & $-0.038(-0.088 ; 0.011)$ & 0.132 & $-0.039(-0.088 ; 0.010)$ & 0.120 \\
\hline FPG (mmol/l) & 916 & $-0.049(-0.131 ; 0.034)$ & 0.249 & $-0.098(-0.180 ;-0.016)$ & 0.020 & $-0.099(-0.182 ;-0.016)$ & 0.020 \\
\hline $2 \mathrm{hPG}(\mathrm{mmol} / \mathrm{l})$ & 601 & $0.100(-0.218 ; 0.417)$ & 0.538 & $-0.060(-0.377 ; 0.258)$ & 0.713 & $-0.139(-0.457 ; 0.179)$ & 0.391 \\
\hline Insulin (pmol/l) & 1001 & $37.713^{\mathrm{b}}(22.326 ; 53.100)$ & $<0.001$ & $25.335(9.922 ; 40.748)$ & 0.001 & $24.751(9.117 ; 40.386)$ & 0.002 \\
\hline HOMA-IR & 875 & $0.834(0.586 ; 1.081)$ & $<0.001$ & $0.537(0.300 ; 0.775)$ & $<0.001$ & $0.496(0.257 ; 0.734)$ & $<0.001$ \\
\hline НОМА- $\beta$ & 875 & $36.922(23.568 ; 50.276)$ & $<0.001$ & $26.141(12.835 ; 39.446)$ & $<0.001$ & $25.024(11.550 ; 38.499)$ & $<0.001$ \\
\hline
\end{tabular}

All results are per doubling of baseline sCD163 concentration

Model 1: Adjusted for age and sex

Model 2: Additionally adjusted for baseline WC

Model 3: Additionally adjusted for baseline smoking and CRP

${ }^{a}$ Example of interpretation: change in insulin is $\mathbf{1 . 0 7 8} \mathrm{pmol} / \mathrm{l}$ per year per doubling of baseline sCD163

${ }^{\mathrm{b}}$ Example of interpretation: the time-independent difference between insulin is $\mathbf{3 7 . 7 1 3}$ pmol/1 by doubling of baseline sCD163 at any time point

associations between $\mathrm{SCD} 163$, adiponectin and CRP per doubling of concentration, and changes per year in $\mathrm{HbA}_{1 \mathrm{c}}$, fasting plasma glucose, $2 \mathrm{~h}$ glucose, fasting insulin, HOMA-IR and HOMA- $\beta$ were assessed using a mixed-effects model.

Results A doubling of sCD163 concentration was positively associated with changes in fasting insulin $(\beta=1.078$ per year, 95\% CI 0.454, 1.702) and HOMA- $\beta(\beta=1.313$ per year, $95 \%$ CI $0.537,2.089$ ), and a doubling of CRP concentration was positively associated with $\mathbf{H b A}_{1 \mathbf{c}}(\beta=\mathbf{0 . 0 0 4}$ per year, $95 \% \mathrm{CI}$ $0.001,0.007)$ and fasting insulin $(\beta=0.267$ per year, $95 \% \mathrm{CI}$ 0.029, 0.504) after adjustment for age and sex. A doubling of adiponectin was inversely associated with changes in fasting glucose $(\beta=-0.017$ per year, 95\% CI $-0.028,-0.005), 2 \mathrm{~h}$ glucose $(\beta=-0.063$ per year, $95 \% \mathrm{CI}-\mathbf{0 . 1 0 7}, \mathbf{- 0 . 0 1 9})$, fasting insulin ( $\beta=-1.558$ per year, 95\% CI $-2.020,-1.096)$, HOMA-IR ( $\beta=\mathbf{- 0 . 0 4 0}$ per year, 95\% CI $-\mathbf{0 . 0 6 2},-\mathbf{0 . 0 1 9})$ and HOMA- $\beta$ ( $\beta=\mathbf{- 1 . 0 0 9}$ per year, $95 \%$ CI $\mathbf{- 1 . 5 8 9},-\mathbf{0 . 4 2 9})$ after adjustment for age and sex. The associations were robust to adjustment for baseline waist circumference and smoking. Adjustment for CRP did not change the associations for sCD163 or adiponectin.

Conclusions/interpretation Our findings indicate that mechanisms related to inflammation, including macrophage activation and adipocyte metabolism, may play a role in changes in glucose homeostasis in individuals at high risk of type 2 diabetes mellitus.

\section{Results}

Study population A total of 2082 (50\%) out of 4188 invited individuals attended, whereas 2106 did not attend the ADDITION-PRO follow-up examination (Fig. 1). We excluded 336 individuals who had developed type 2 diabetes mellitus during follow-up. These individuals had higher levels of sCD163 (1.8 vs $1.6 \mathrm{mg} / 1, p<0.001)$ and CRP (25.7 vs $16.2 \mathrm{nmol} / \mathrm{l}, p<0.001)$ and lower levels of adiponectin (11.5 vs $14.4 \mu \mathrm{g} / \mathrm{ml}, p<0.001$ ) at baseline compared with individuals who did not develop type 2 diabetes during follow-up. We further excluded 667 individuals who did not have baseline biobank materials. Descriptive characteristics of the individuals with and without biobank materials are shown in ESM Table 1. We additionally excluded 65 individuals with CRP concentrations above $95 \mathrm{nmol} / \mathrm{l}$, taken as evidence of acute infection.

Thus, 1014 individuals were eligible for the present study. Descriptive characteristics of the study population including median levels of the biomarkers are shown in Table 1. Higher levels of adiponectin $(17.9 \mathrm{vs} 12.1 \mu \mathrm{g} / \mathrm{ml}, p<0.001)$ and CRP ( 19.0 vs $12.4 \mathrm{nmol} / 1, p<0.001)$ were found in women than men. No sex differences were found in the level of sCD163. The mean duration of follow-up was $7.5(\mathrm{SD}=1.4)$ years.

Changes in glycaemia, insulin resistance and insulin secretion during follow-up $\mathrm{HbA}_{1 \mathrm{c}}$ and $\mathrm{FPG}$ concentrations 
Table 3 Association of baseline adiponectin with glycaemia, insulin resistance and insulin secretion

\begin{tabular}{|c|c|c|c|c|c|c|c|}
\hline \multirow[t]{2}{*}{ Outcome } & \multirow[t]{2}{*}{$n$} & \multicolumn{2}{|l|}{ Model 1} & \multicolumn{2}{|l|}{ Model 2} & \multicolumn{2}{|l|}{ Model 3} \\
\hline & & $\beta(95 \% \mathrm{CI})$ & $p$ value & $\beta(95 \% \mathrm{CI})$ & $p$ value & $\beta(95 \% \mathrm{CI})$ & $p$ value \\
\hline \multicolumn{8}{|l|}{ Time-dependent } \\
\hline $\mathrm{HbA}_{1 \mathrm{c}}(\%)$ & 965 & $-0.003(-0.008 ; 0.002)$ & 0.171 & $-0.003(-0.008 ; 0.002)$ & 0.179 & $-0.004(-0.009 ; 0.002)$ & 0.170 \\
\hline FPG (mmol/l) & 913 & $-0.017^{\mathrm{a}}(-0.028 ;-0.005)$ & 0.004 & $-0.017(-0.028 ;-0.006)$ & 0.003 & $-0.017(-0.028 ;-0.006)$ & 0.003 \\
\hline $2 \mathrm{hPG}(\mathrm{mmol} / \mathrm{l})$ & 600 & $-0.063(-0.107 ;-0.019)$ & 0.005 & $-0.062(-0.106 ;-0.017)$ & 0.006 & $-0.062(-0.106 ;-0.017)$ & 0.006 \\
\hline Insulin (pmol/1) & 998 & $-1.558(-2.020 ;-1.096)$ & $<0.001$ & $-1.557(-2.019 ;-1.094)$ & $<0.001$ & $-1.557(-2.019 ;-1.094)$ & $<0.001$ \\
\hline HOMA-IR & 872 & $-0.040(-0.062 ;-0.019)$ & $<0.001$ & $-0.041(-0.062 ;-0.020)$ & $<0.001$ & $-0.041(-0.062 ;-0.019)$ & $<0.001$ \\
\hline НОМА- $\beta$ & 872 & $-1.009(-1.589 ;-0.429)$ & 0.001 & $-1.017(-1.597 ;-0.437)$ & 0.001 & $-1.017(-1.597 ;-0.437)$ & 0.001 \\
\hline \multicolumn{8}{|l|}{ Time-independent } \\
\hline $\mathrm{HbA}_{1 \mathrm{c}}(\%)$ & 965 & $-0.033(-0.073 ; 0.007)$ & 0.107 & $-0.018(-0.058 ; 0.022)$ & 0.384 & $-0.006(-0.046 ; 0.034)$ & 0.751 \\
\hline FPG (mmol/l) & 913 & $-0.029(-0.095 ; 0.036)$ & 0.382 & $0.008(-0.058 ; 0.074)$ & 0.803 & $0.011(-0.055 ; 0.077)$ & 0.741 \\
\hline $2 \mathrm{hPG}(\mathrm{mmol} / \mathrm{l})$ & 600 & $-0.097(-0.356 ; 0.163)$ & 0.465 & $0.035(-0.224 ; 0.294)$ & 0.792 & $0.062(-0.196 ; 0.320)$ & 0.639 \\
\hline Insulin $(\mathrm{pmol} / \mathrm{l})$ & 998 & $-29.604^{\mathrm{b}}(-42.555 ;-16.652)$ & $<0.001$ & $-19.748(-32.681 ;-6.815)$ & 0.003 & $-19.975(-32.988 ;-6.963)$ & 0.003 \\
\hline HOMA-IR & 872 & $-0.549(-0.753 ;-0.346)$ & $<0.001$ & $-0.303(-0.497 ;-0.109)$ & 0.002 & $-0.275(-0.468 ;-0.081)$ & 0.005 \\
\hline HOMA- $\beta$ & 872 & $-19.340(-30.423 ;-8.257)$ & 0.001 & $-9.949(-20.954 ; 1.057)$ & 0.076 & $-9.246(-20.297 ; 1.805)$ & 0.101 \\
\hline
\end{tabular}

All results are per doubling of baseline adiponectin concentration

Model 1: Adjusted for age and sex

Model 2: Additionally adjusted for baseline WC

Model 3: Additionally adjusted for baseline smoking and CRP

${ }^{a}$ Example of interpretation: change in $\mathbf{F P G}$ is $\mathbf{- 0 . 0 1 7} \mathbf{~ m m o l} / \mathrm{l}$ per year per doubling of baseline adiponectin

${ }^{\mathrm{b}}$ Example of interpretation: the time-independent difference between insulin is $\mathbf{- 2 9 . 6 0 4} \mathrm{pmol} / \mathrm{l}$ by doubling of baseline adiponectin at any time point

increased during follow-up after age and sex adjustment: $\mathbf{0 . 0 0 8 \%}$ (95\% CI $\mathbf{0 . 0 0 5}, \mathbf{0 . 0 1 2} ; \mathbf{0 . 0 8 8} \mathrm{mmol} / \mathrm{mol}, 95 \%$ CI $\mathbf{0 . 0 5 1}, \mathbf{0 . 1 2 6})$ per year for $\mathrm{HbA}_{1 \mathrm{c}}$ and $\mathbf{0 . 0 6 3} \mathrm{mmol} / \mathrm{l}(95 \% \mathrm{CI}$ $\mathbf{0 . 0 5 5}, \mathbf{0 . 0 7 1}$ ) per year for FPG. . The estimates for yearly rates of increase were further strengthened after accounting for baseline levels of each respective variable (ESM Table 2). Fasting insulin, HOMA-IR and HOMA- $\beta$ values decreased during follow-up after adjustment for age and sex: $\mathbf{- 4 . 3 5 7}$ $\mathrm{pmol} / 1(95 \% \mathrm{CI} \mathbf{- 4 . 6 7 9}, \mathbf{- 4 . 0 3 5})$ per year for fasting insulin, $\mathbf{- 0 . 0 5 4}(95 \%$ CI $-\mathbf{0 . 0 6 9},-\mathbf{0 . 0 4 0})$ per year for HOMA-IR and $\mathbf{- 6 . 2 7 8}(95 \%$ CI $\mathbf{- 6 . 6 7 8}, \mathbf{- 5 . 8 7 8})$ per year for HOMA- $\beta$. These decreases were steeper on inclusion of baseline insulin levels (ESM Table 2). Further adjustment for baseline WC and smoking did not materially change the estimates, and estimates remained statistically significant. No significant changes were found for $\mathbf{2 h P G}$.

Baseline sCD163, adiponectin, CRP and changes in glycaemia, insulin resistance and insulin secretion Baseline sCD163 and CRP were positively associated with changes in fasting insulin, and in addition, $\mathrm{SCD} 163$ was positively associated with changes in HOMA- $\beta$ and CRP was positively associated with changes in $\mathbf{H b A}_{1 \mathrm{c}}$ after adjustment for age and sex. Baseline adiponectin was negatively associated with changes in FPG, 2hPG, fasting insulin,
HOMA-IR and HOMA- $\beta$ after age and sex adjustment (Tables 2, 3 and 4). In contrast to adiponectin, sCD163 and CRP were not significantly associated with changes in FPG, 2hPG or HOMA-IR. Baseline sCD163, and adiponectin were not associated with any statistically significant changes in $\mathrm{HbA}_{1 \mathrm{c}}$. The associations remained stable after further adjustment for baseline WC. In addition, adjustment for baseline smoking status and CRP did not change estimates for sCD163 and adiponectin. Additional adjustment for smoking did not materially change the estimates for CRP. No sex differences were found in analysis of any of the markers.

Baseline sCD163, adiponectin, CRP and time-independent associations with glucose and insulin variables Baseline sCD163 and CRP showed a positive, and adiponectin a negative, time-independent association with fasting insulin, HOMA-IR and HOMA- $\beta$ after adjustment for age and sex (Tables 2, 3 and 4). In addition, CRP showed a positive time-independent association with $\mathbf{H b A}_{1 \mathrm{c}}$ and $2 \mathrm{hPG}$. The significant estimates were markedly attenuated by $29-\mathbf{3 6 \%}$ for $\mathrm{sCD} 163$, by $\mathbf{3 3}-\mathbf{4 9 \%}$ for adiponectin and by $\mathbf{2 9 - 5 7 \%}$ for CRP after additional adjustment for WC. All estimates remained statistically significant except for the association between 
Table 4 Association of CRP with glycaemia, insulin resistance and insulin secretion

\begin{tabular}{|c|c|c|c|c|c|c|c|}
\hline \multirow[t]{2}{*}{ Outcome } & \multirow[t]{2}{*}{$n$} & \multicolumn{2}{|l|}{ Model 1} & \multicolumn{2}{|l|}{ Model 2} & \multicolumn{2}{|l|}{ Model 2b } \\
\hline & & $\beta(95 \% \mathrm{CI})$ & $p$ value & $\beta(95 \% \mathrm{CI})$ & $p$ value & $\beta(95 \% \mathrm{CI})$ & $p$ value \\
\hline \multicolumn{8}{|l|}{ Time-dependent } \\
\hline $\mathrm{HbA}_{1 \mathrm{c}}(\%)$ & 968 & $0.004^{\mathrm{a}}(0.001 ; 0.007)$ & 0.010 & $0.004(0.001 ; 0.007)$ & 0.010 & $0.004(0.001 ; 0.007)$ & 0.009 \\
\hline $\mathrm{FPG}(\mathrm{mmol} / \mathrm{l})$ & 916 & $0.004(-0.003 ; 0.010)$ & 0.235 & $0.004(-0.002 ; 0.010)$ & 0.218 & $0.004(-0.002 ; 0.010)$ & 0.219 \\
\hline $2 \mathrm{hPG}(\mathrm{mmol} / \mathrm{l})$ & 601 & $0.000(-0.026 ; 0.026)$ & 0.994 & $-0.001(-0.026 ; 0.025)$ & 0.955 & $-0.001(-0.026 ; 0.025)$ & 0.955 \\
\hline Insulin (pmol/l) & 955 & $0.267(0.029 ; 0.504)$ & 0.028 & $0.270(0.032 ; 0.508)$ & 0.026 & $0.270(0.032 ; 0.508)$ & 0.026 \\
\hline HOMA-IR & 875 & $0.009(-0.003 ; 0.022)$ & 0.146 & $0.010(-0.003 ; 0.022)$ & 0.139 & $0.010(-0.003 ; 0.022)$ & 0.140 \\
\hline НOMA- $\beta$ & 875 & $0.328(-0.015 ; 0.672)$ & 0.061 & $0.330(-0.013 ; 0.674)$ & 0.059 & $0.330(-0.013 ; 0.674)$ & 0.059 \\
\hline \multicolumn{8}{|l|}{ Time-independent } \\
\hline $\mathrm{HbA}_{1 \mathrm{c}}(\%)$ & 968 & $0.028^{b}(0.006 ; 0.049)$ & 0.011 & $0.018(-0.005 ; 0.040)$ & 0.124 & $0.012(-0.011 ; 0.034)$ & 0.308 \\
\hline FPG (mmol/l) & 916 & $0.020(-0.016 ; 0.057)$ & 0.278 & $-0.009(-0.047 ; 0.028)$ & 0.617 & $-0.011(-0.049 ; 0.026)$ & 0.550 \\
\hline $2 \mathrm{hPG}(\mathrm{mmol} / \mathrm{l})$ & 601 & $0.284(0.146 ; 0.421)$ & $<0.001$ & $0.202(0.061 ; 0.343)$ & 0.005 & $0.204(0.063 ; 0.345)$ & 0.005 \\
\hline Insulin (pmol/l) & 955 & $7.739(5.314 ; 10.164)$ & $<0.001$ & $3.507(1.132 ; 5.883)$ & 0.004 & 3.507 (1.118; 5.896) & 0.004 \\
\hline HOMA-IR & 875 & $0.326(0.216 ; 0.435)$ & $<0.001$ & $0.146(0.039 ; 0.254)$ & 0.007 & $0.143(0.035 ; 0.251)$ & 0.009 \\
\hline НОМА- $\beta$ & 875 & $11.959(6.032 ; 17.886)$ & $<0.001$ & $5.180(-0.852 ; 11.212)$ & 0.092 & $5.214(-0.855 ; 11.283)$ & 0.092 \\
\hline
\end{tabular}

All results are per doubling of baseline CRP concentration

Model 1: Adjusted for age and sex

Model 2: Additionally adjusted for baseline WC

Model 2b: Additionally adjusted for baseline smoking

${ }^{\text {a }}$ Example of interpretation: change in $\mathbf{H b A}_{\mathbf{1 c}}$ is $\mathbf{0 . 0 0 4 \%}$ per year per doubling of baseline CRP

${ }^{\mathrm{b}}$ Example of interpretation: the time-independent difference between $\mathbf{H b A}_{\mathbf{1 c}}$ is $\mathbf{0 . 0 2 8 \%}$ by doubling of baseline CRP at any time point

adiponectin and HOMA- $\beta$, as well as the association between CRP and both $\mathrm{HbA}_{1 \mathrm{c}}$ and HOMA- $\beta$. In addition, SCD163 showed a negative association with FPG after WC adjustment. Estimates for sCD163 and adiponectin changed minimally after additional adjustment for CRP and remained statistically significant.

Analyses of effect modification by sex showed that although there was no statistically significant overall or sexspecific time-independent association between sCD163 and $2 \mathrm{hPG}$, the magnitude of the effect estimate differed by sex: $\beta_{\text {women }}=\mathbf{- 0 . 2 4 5}(95 \%$ CI $\mathbf{- 0 . 6 9 4}, \mathbf{0 . 2 0 4}) ; \beta_{\text {men }}=\mathbf{0 . 4 3 2}$, $(95 \%$ CI $\mathbf{- 0 . 0 1 4}, \mathbf{0 . 8 7 9})\left(p_{\text {interaction }}=\mathbf{0 . 0 0 6}\right)$. In addition, women had a less pronounced time-independent association between $\mathrm{sCD} 163$, and HOMA-IR than men $\left(\beta_{\text {women }}=\mathbf{0 . 4 5 8}\right.$, $95 \%$ CI $\mathbf{0 . 0 8 7}, \mathbf{0 . 8 2 9} ; \beta_{\text {men }}=\mathbf{1 . 1 0 5}, 95 \%$ CI $\mathbf{0 . 7 7 0}, \mathbf{1 . 4 4 0}$; $\left.\mathrm{p}_{\text {interaction }}=0.020\right)$, and FPG than men $\left(\beta_{\text {women }}=-0.095\right.$, 95\% CI $-0.217,0.026 ; \beta_{\text {men }}=-0.010,95 \%$ CI -0.123 , 0.102; $p_{\text {interaction }}=\mathbf{0 . 0 2 8}$ ). No statistically significant effect modification by sex was found for any of the other analyses.

\section{Discussion}

We found markers of inflammation and adipocyte metabolism to be associated with metabolic changes in glycaemia, insulin resistance and insulin secretion among individuals at high risk of diabetes. sCD163 was positively associated with subsequent changes in insulin secretion, whilst both sCD163 and CRP were associated with fasting insulin. In contrast, adiponectin was inversely associated with changes in fasting glucose, post-load glucose, fasting insulin, insulin resistance and insulin secretion. The associations were robust to adjustment for potential confounders. We also found that sCD163, CRP and adiponectin showed time-independent associations with fasting insulin and insulin resistance, and additionally that $\mathrm{SCD} 163$ exhibited a time-independent association with fasting glucose (only when also adjusted for baseline WC, smoking and CRP) and insulin secretion, and CRP with post-load glucose. Our results may reflect the metabolic changes in fasting and post-load glucose, insulin resistance and insulin secretion found in high-risk individuals in the years before the onset of type 2 diabetes mellitus [19].

An increase in insulin resistance, accompanied by a compensatory increase in insulin secretion, is often one of the first abnormal features marking the transition between normal glucose tolerance and a trajectory leading to diabetes. It is only when this compensatory mechanism fails that levels of first post-load glucose, and later also fasting glucose, start to rise [19]. With this model in mind, our finding of an association between adiponectin and changes in fasting glucose, postload glucose, as well as insulin resistance and insulin secretion, may be interpreted as indicating, that adiponectin is 
linked to a broader set of mechanisms and a wider time window in the pathogenesis of dysglycaemic progression than are sCD163 and CRP, which in our study were only associated with changes in insulin secretion (sCD163 only) and fasting insulin, and not with changes in insulin resistance or any of the glucose variables. Our findings support the hypothesis that inflammation may be involved in the pathogenesis of dysglycaemic progression in high-risk individuals and the idea that markers of inflammation and adipocyte metabolism and their specific role may vary at different stages.

The present study is to our knowledge the first to investigate the association between $\mathrm{SCD} 163$ and changes in glucose and insulin variables. A previous prospective study found an association between sCD163 and risk of incident type 2 diabetes mellitus in the general population during 18 years of follow-up independently of age, BMI and CRP [10]. Our findings strengthen and deepen this notion by documenting the association between SCD163 and changes in insulin secretion in individuals at high risk of diabetes. The relations remained statistically significant after adjustment for CRP, indicating that systemic low-grade inflammation does not explain the effect of sCD163 on changes in insulin secretion. We did not find any association to glucose variables, presumably because increased fasting and post-load glucose are relatively late events in the pathogenesis of dysglycaemic progression, and because participants with diabetes diagnosed during follow-up were excluded by our study design. Our finding of time-independent associations between SCD163 and insulin resistance and insulin secretion strengthens the evidence from previous cross-sectional studies [7, 20].

Our study is in agreement with other studies that found adiponectin to be negatively associated with changes in fasting glucose, post-load glucose, insulin resistance and insulin secretion [21-23]. In the ADDITION-PRO study, we did not find any associations linking adiponectin with changes in $\mathrm{HbA}_{1 \mathrm{c}}$, as reported in previous studies after adjustment for WC, lipids [24] and baseline fasting glucose [25]. The levels of adiponectin in the above-mentioned studies were $34-55 \%$ lower than the adiponectin level in our study and may primarily be due to the different adiponectin assays used. Although the adiponectin levels in our study are higher, any linear difference between the results of different assays is unlikely to bias the relative measures of association we report. The relationships in our study were unaffected by adjustment for CRP, indicating that systemic low-grade inflammation does not confound the association between either adiponectin or sCD163 and measures of dysglycaemia.

We found that CRP levels were positively associated with changes in $\mathbf{H b A}_{\mathbf{1 c}}$ and fasting insulin, but not with any changes in insulin resistance or other glucose variables. This is supported by one previous study that found baseline CRP to be associated with changes in $\mathrm{HbA}_{1 \mathrm{c}}$ during 7 years of follow-up [24] and in contrast to another study that found baseline CRP to be associated with changes in insulin resistance during 5 years of follow-up [26]. The different study populations may explain the differences as we included individuals with a high risk of diabetes, while the previous study examined a study sample without specific selection for elevated diabetes risk. Since CRP increases less steeply over time among individuals who develop type 2 diabetes mellitus compared with controls [27], our study population may have had a less steep CRP trajectory than populations in previous studies, possibly leading to different results.

None of the previous studies examined time-independent associations, which may also lead to different results. We investigated the time-dependent and time-independent association between the biomarkers and our outcomes in a single mixed-effects model that allowed us to estimate the separate effects for the time-independent and time-dependent associations simultaneously. The time-independent estimates in our model can be compared with cross-sectional analyses, while the time-dependent estimates can be compared with analyses of change. There are, however, some important differences: time-independent outcomes refer to interindividual differences in the level of the outcome, at any age. Timedependent outcomes refer to interindividual differences in the velocity of change as people age. In traditional crosssectional analyses, the outcome is not only determined by contemporaneous (time-independent) mechanisms around the time of assessment, but also by the sum of timedependent effects in the past. Our analysis approach separates these two components by regarding both baseline and followup measurements as expressions of the same system, at two different points in time.

Obesity, in particular abdominal obesity, is associated with low-grade inflammation and type 2 diabetes mellitus [2]. Thus, in studies of associations between low-grade inflammation and type 2 diabetes mellitus, it is important to take obesity into account as a confounding factor. As WC estimates abdominal obesity rather than total fat mass, we adjusted for WC instead of BMI. We observed that the time-independent associations were markedly reduced after adjustment for baseline WC. This phenomenon was not observed in the timedependent associations. Our findings indicate that central obesity, by causing some degree of low-grade inflammation as well as insulin resistance, partially confounds the associations between low-grade inflammation and the levels of different measures of dysglycaemia. However, it does not attenuate the association between low-grade inflammation and the velocity of dysglycaemic progression.

Our study has several strengths. First, it is among the first studies to assess SCD163, adiponectin and CRP and the longitudinal relationship with subsequent changes in different glucose and insulin variables using mixed-effects models, thereby estimating time-independent and time-dependent associations in the same model. Second, our large study 
population, its representation of all relevant dysglycaemic risk levels and long follow-up period allowed us to home in on precisely the pathophysiological time window where the effects under study are most likely to play out and to describe these processes in detail.

The main limitation of our study is the different assays and methods used to quantify glucose and insulin at baseline and follow-up. Baseline glucose was measured in capillary blood, whereas follow-up glucose was measured in venous blood. Most previous studies found consistently higher glucose concentrations in capillary blood than venous blood [28-30]. As a consequence, we may have underestimated the absolute differences in fasting and post-load glucose levels, and the associations between the biomarkers and changes in glucose variables. Insulin concentrations were quantified using a different insulin assay at baseline and follow-up. Serum insulin may vary by a factor of two for median and maximum value using different assays [31]. To meet this challenge, we compared the two different assays by re-analysing follow-up insulin levels using the baseline methods. As we found a difference between insulin levels measured by the two methods, we applied a correction factor to our baseline insulin values.

Although we found associations between the biomarkers and the longitudinal change in different glucose and insulin variables, and we adjusted for relevant confounders, we cannot conclude that these associations contribute causally to the development of type 2 diabetes mellitus. First, we did not have the possibility to examine changes in glucose and insulin variables among individuals who developed type 2 diabetes mellitus during the follow-up period. As a result the influence of the biomarkers was only investigated in individuals at high risk of type 2 diabetes mellitus. Second, as in any observational epidemiological study, we cannot fully exclude reverse causation or residual confounding as explanations for our findings. Mendelian randomisation studies are needed to elucidate the causality between biomarkers of low-grade inflammation and the metabolic changes leading to type 2 diabetes mellitus. The causality of the association between adiponectin and insulin resistance and type 2 diabetes mellitus has previously been investigated in Mendelian randomisation studies, with diverging results [32, 33], while a similar study for CRP found that its relation to insulin resistance, glycaemia and type 2 diabetes mellitus was probably non-causal [34]. No Mendelian randomisation studies to date have examined the causality of the association between sCD163 and type 2 diabetes mellitus.

In conclusion, our 7 year follow-up study demonstrates that sCD163 was positively associated with insulin secretion and both sCD163 and CRP were associated with fasting insulin, whereas adiponectin was inversely associated with changes in fasting glucose, post-load glucose, fasting insulin, insulin resistance and secretion in a population of individuals at high risk of diabetes independently of baseline abdominal obesity. Furthermore, associations of sCD163 and adiponectin were independent of systemic low-grade inflammation. Our findings indicate that mechanisms related to macrophage activation and adipocyte metabolism may play a role in changes of glucose homeostasis in individuals at high risk of type 2 diabetes mellitus. 